\title{
A microscopic approach to studying colloidal stability
}

\author{
Zhiwei Sun ${ }^{\text {a) }}$ \\ Open Laboratory of Bond Selective Chemistry, University of Science and Technology of China, Hefei, \\ 230026, Anhui, People's Republic of China, and National Microgravity Lab, Institute of Mechanics, Chinese \\ Academy of Sciences, Beijing 100080, People's Republic of China \\ Shenghua $\mathrm{Xu}$ \\ Open Laboratory of Bond Selective Chemistry, University of Science and Technology of China, Hefei, \\ 230026, Anhui, People's Republic of China \\ Guoliang Dai \\ National Microgravity Lab, Institute of Mechanics, Chinese Academy of Sciences, Beijing 100080, \\ People's Republic of China \\ Yinmei Li and Liren Lou \\ Open Laboratory of Bond Selective Chemistry, University of Science and Technology of China, Hefei, \\ 230026, Anhui, People's Republic of China \\ Qiusheng Liu \\ National Microgravity Lab, Institute of Mechanics, Chinese Academy of Sciences, Beijing 100080, \\ People's Republic of China \\ Ruzeng Zhu \\ LNM, Institute of Mechanics, Chinese Academy of Sciences, Beijing 100080, People's Republic of China
}

(Received 19 February 2003; accepted 1 May 2003)

A novel approach to the investigation of colloidal aggregation in experiments performed at microscopic particle levels by means of artificially induced particle collisions with the aid of optical tweezers is proposed. A physical model describing the artificially induced collisions was suggested. The stability ratios for different electrolyte concentrations $(\mathrm{NaCl})$ are estimated by dividing the total number of particle collisions by the number of collisions leading to permanent doublets. The experimental results under different electrolyte concentrations are compared with zeta potentials and turbidity measurements. In regards to fully dispersed or rapid-coagulated systems, this approach can conveniently provide an accurate judgment of the system's stability. For general slow-coagulated systems, when the stability ratio is greater than 1.8 , our data are in agreement with those obtained from the turbidity measurements. When the stability ratio is less than 1.8 , the accuracy of the approach is limited due to the possibility of the imprecise control of the short duration during which the particle pair is held in the optical trap for the induced collisions. (C) 2003 American Institute of Physics. [DOI: 10.1063/1.1585022]

\section{INTRODUCTION}

All the characteristics of colloidal systems change remarkably in the transition from the dispersed to the aggregated state. Even within aggregated systems, the degree of aggregation varies tremendously. The question of how to determine the state of colloidal systems is of central importance to predicting and controlling the stability of colloidal suspensions. The stability ratio $W$ is typically used to describe the degree of stability of a colloidal system. The stability ratio $W$ is commonly estimated by the ratio of the diffusioncontrolled rapid coagulation rate $\left(k_{\text {rapid }}\right)$ to the coagulation rate $(k)$ of the system under consideration: ${ }^{1-4}$

$W=k_{\text {rapid }} / k$.

Commonly, turbidity measurements, ${ }^{5-9}$ low angle light

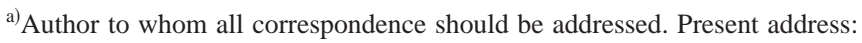
National Microgravity Lab, Institute of Mechanics, Chinese Academy of Sciences, Beijing 100080, P. R. China; Electronic mail: sunzw@ imech.ac.cn
}

scattering, ${ }^{10-13}$ and dynamic light scattering ${ }^{14-17}$ are used to determine the coagulation rates and therefore the stability ratio. The methods based on Eq. (1) require information on the long-term, accumulated effects of the motions and interactions of huge numbers of particles; thus, we can reasonably call these a "macroscopic approach."

On the other hand, $W$ is physically equal to the ratio of the total number of particle collisions $(n)$ to the number of collisions leading to permanent doublets $\left(n_{c}\right):^{3,4}$

$$
W=n / n_{c} .
$$

The reciprocal of the stability ratio $1 / W$ is the so-called "sticking probability" or "collision efficiency," $p=n_{c} / n \cdot{ }^{18-20}$ For slow coagulation [reaction limited cluster aggregation (RLCA)], the energy barrier prevents every encounter from effective aggregation. Only a fraction, $1 / W$, of collisions are successful. The features of colloidal aggregation are determined by particle collisions and interactions. Therefore a more insightful approach to obtaining the stability ratio would be to directly examine particle collisions and 
to check the percentage of colliding particles that stick together. Due to the random nature of particle movement, however, one is unable to locate where a collision takes place; therefore such a microscopic observation can hardly be implemented. To our knowledge, no one has experimentally evaluated the stability of a colloidal system based on Eq. (2). In this study, we present a new methodology with the use of optical tweezers ${ }^{21-24}$ to make the above-mentioned microscopic observation possible to the study of colloidal stability at individual particle levels.

This paper is organized as follows: In Sec. II, we describe the basic consideration and feasibility regarding this new approach. Section III presents our experimental arrangement. Section IV shows our experimental results, interpretation, and comparisons along with data obtained from other approaches (including the turbidity and zeta potential measurements). Section V contains our conclusion.

\section{ANALYSIS}

Optical tweezers (or optical trap) ${ }^{21-24}$ are a very useful tool for manipulation of small particles without influencing the particles and their surrounding medium. It can be designed compactly and has the advantage that a single microscope can be used to trap and view particles at the same time. Brownian motions make particle collisions take place at unpredictable locations; therefore one has no way to employ a microscope to investigate particle collisions and its outcomes. If we use optical tweezers to catch two particles and bring them together for a collision, we would be able to confine their collision in an area observable by a microscope. Observing this "artificially induced collision," it is possible to check how particle pairs interact with each other and what occurs after their release from the trap: whether they stick together or separate. In contrast with traditional methods of studying colloidal stability using Eq. (1), we attempt a step toward understanding the colloidal stability by means of a microscopic approach based on Eq. (2), through the observation of artificially arranged collisions.

Imagine, when two particles are restrained in the optical trap for a certain duration, what would happen after they are released. Apparently, if the interaction energy barrier opposing coagulation disappears, two particles trapped in the optical trap would keep sticking together after they are released. Actually when two (uncharged) particles approach each other to a certain distance, the van der Waals attraction will pull the particles together for coagulation. On the other hand, if the potential barrier is very high (highly stable dispersions) in comparison with the particle thermal energy $\left(k_{B} T\right)$, (where $k_{B}$ is the Boltzmann constant and $T$ is temperature), the particle pair in the trap will separate upon release. Therefore, a test of the "artificially induced collision" will allow us to easily make a judgment regarding whether a system is in a highly dispersed or rapid-coagulated status.

For slow coagulations, however, it is impossible to make predictions regarding the adhesion between the two particles by one single test of our artificially induced collision described earlier. It needs to perform a series of such tests to collect enough information for deducing the stability of a system using Eq. (2). We will analyze this case in the following.

Consider two particles that are caught into the optical trap at the time $t=0$ and released at the time $t=\tau$. For convenience, we will refer to this holding time as $\tau$ in the trap as the "trapping duration" hereafter. Let $P(\tau)$ be the total (or accumulated) sticking probability for the particle pair to stick together after being released from the trap at the time $=\tau$ [then we have $(1-P)$ to be the probability of the particle still being apart at time $t=\tau] ; p$ the sticking probability after a single collision of a particle pair in the optical trap, and $f$ the collision frequency in the trap. Assuming the coagulation is not reversible, we have the following relationship:

$$
\begin{aligned}
& d(1-P)=-(1-P) p f d t, \\
& \ln (1-P)=(-p f) t, \\
& P=1-e^{-p f t} . \\
& \text { When } p f t \ll 1, \text { we have }
\end{aligned}
$$

$$
P \approx p f t \text {. }
$$

We can see that $\ln (1-P)$ is linearly related to time and its slope is $p f$. In this case if the collision frequency is known, we can obtain the sticking probability $p$ and therefore the stability ratio $W(=1 / p)$. However, it is impractical to evaluate the collision frequency of the particle pair in the trap with accuracy, because it is hard to measure the actual volume and the shape confined by the trap. In addition, collision frequencies may vary with the nature of particle interaction.

For rapid coagulations, there is no repulsive force between particles; in fact, the van der Waals attraction will even pull particles together for coagulation. Thus, the collision frequency is high. For slow coagulations, however, repulsive force attempts to push particles apart so that particles are accommodated to an extended area, resulting in lower collision frequency. As for the coagulation reaction, only when the kinetic energy associated with the velocity component on the line connecting centers of the two particles is large enough to overcome the potential barrier between particles, they become coagulated.

A major difference between our artificially induced collisions and natural ones is that at the beginning when two particles are hauled into the optical trap for collision (see the procedure for the artificially induced collision described in Sec. III) there is an additional directed head-on speed, which we will call the "trapping speed" hereafter. If this "trapping speed" were compatible with or greater than their thermal speeds, the measurement regarding the adhesion between the two particles would not be applicable to the real situation. The average kinetic energy of a particle is $\frac{1}{2} m v^{2}=\frac{3}{2} k_{B} T$, where $m, v, k_{B}$ and $T$ are the mass, velocity of the particle, Boltzmann constant and temperature, respectively. For the system we are considering, the average thermal speed of the particles is about $0.5 \mathrm{~cm} \mathrm{~s}^{-1}$ (at $20^{\circ} \mathrm{C}$ ). According to our observations, the trapping speed is estimated to be less than $0.03 \mathrm{~mm} \mathrm{~s}^{-1}$. Therefore, direct influence of the trapping speed should not be a significant factor in the nature of collisions caused by thermal motions. However, the trapping 
speed may considerably contribute to the collision frequency. Two particles in the trap have a tendency to stay apart due to the repulsion, but the trapping speed makes them approach each other.

A particle pair in the trap would experience two different statuses. We call the first one "compact status;" that is, at the beginning when the second particle is pulled into the trap, the head-on impact makes the particle pair stay closer. And then after the impact speed dampens down, they become relaxed and stay in an extended room-we call this the "relaxed status." Apparently, when two particles are compressed into a small volume-as in the compact status, they would have a higher probability of colliding than in a larger volume - as in the relaxed status. Therefore, the "trapping speed" may lead to a significant raise in the sticking probability due to higher collision frequency at the beginning of the artificially induced collision.

Considering that collision frequencies are different when particle pairs are in the compact status and in the relaxed one, if we use $P(\tau)$ to denote the accumulated sticking probability during the trapping duration $\tau$, we would have

$$
P(\tau)=p f_{c} \tau_{1}+p f_{r}\left(\tau-\tau_{1}\right),
$$

where $\tau_{1}$ is the duration for which the compact status lasts, $f_{c}$ and $f_{r}$ are the collision frequencies in the compact and relaxed statuses, respectively. " $p$ " should keep the same in the both statuses. Since $f_{c} \gg f_{r}$, the sticking probability $P(\tau)$ is primarily determined by the behavior of particle interaction in the compact status, as long as $p$ is small and $\tau$ is not too long.

A Monte Carlo simulation ${ }^{25}$ using a hard sphere model has demonstrated that the collision frequency of two particles is around $10^{3} / \mathrm{s}$ in an order of magnitude when their separation range is limited to $0.001 \mu \mathrm{m}$. It also shows that collision frequency drops down very quickly with increase of their separation. For the hard sphere model, there is no coagulation involved so the particle pair keeps colliding and bounced. Compared with the hard sphere model, in our case repulsion between particles should lower the collision frequency by a couple of orders in magnitude and cause the decline of collision frequency more rapidly as the separation increases.

Now we will further show the possibility that only a single collision actually takes place to a particle pair in the compact status. When the trapping speed brings a particle pair to come into the compact status, the two particles will experience at least one collision due to very high collision frequency there. As a result of this collision, there will be only two outcomes possible: either they will stick together or rebound apart. If they stick together, as an irreversible coagulation there will be no more collisions (only a doublet left) for a two-particle system. If they rebound apart, the backward velocity and the repulsion will drive them away from the compact status rapidly to enter into the relaxed status before any additional effective collision occurs. Therefore, our deduction is that the particle pair will collide once within the compact status, and only once. If this assumption holds, it means $f_{c} \tau_{1} \approx 1$. How long the $\tau_{1}$ lasts will depend on the time for the first collision to take place. It should be

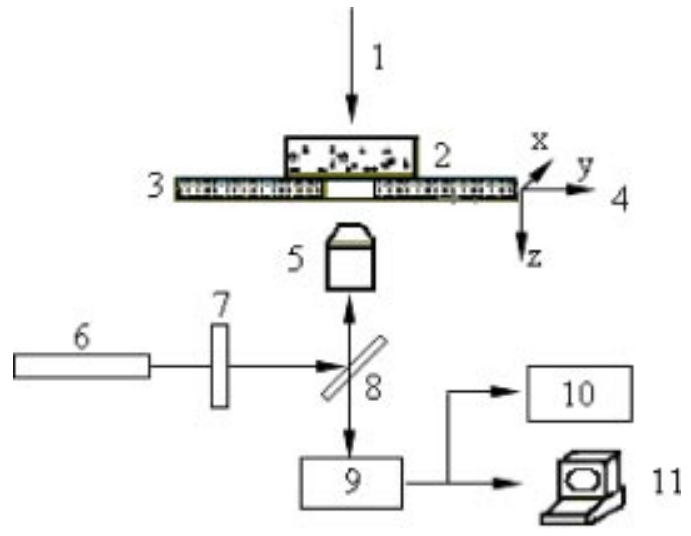

FIG. 1. Experimental setup. 1-illumination light; 2-sample cell; 3-sample stage; 4-3D motion; 5-objective; 6-light source of optical tweezers; 7-electronic shutter; 8-dichromatic mirror; 9-CCD camera; 10 -video recorder; 11 -computer.

very short because of the high collision frequency in the compact status. When $\tau \gg \tau_{1}$, as an approximation of Eq. (7), we will have

$$
p \approx P(\tau)-p f_{r} \tau,
$$

where $p f_{r}$ can be evaluated using Eq. (7) with $\tau \gg \tau_{1}$, as an approximation, from $\left(P\left(\tau^{\prime \prime}\right)-P\left(\tau^{\prime}\right)\right) /\left(\tau^{\prime \prime}-\tau^{\prime}\right)$. Here $P\left(\tau^{\prime \prime}\right)$ and $P\left(\tau^{\prime}\right)$ are the accumulated sticking probabilities, under the relaxed status, for the trapping duration at $\tau=\tau^{\prime}$ and $\tau$ $=\tau^{\prime \prime}$, respectively. So we have

$$
p \approx P(\tau)-\left(P\left(\tau^{\prime \prime}\right)-P\left(\tau^{\prime}\right)\right) t /\left(\tau^{\prime \prime}-\tau^{\prime}\right) .
$$

If we take $\tau^{\prime}=1 \mathrm{~s}$ and $\tau^{\prime \prime}=2 \mathrm{~s}$, we will finally have

$$
p \approx 2 P(\tau=1)-P(\tau=2) \text {. }
$$

\section{EXPERIMENTAL ARRANGEMENT}

To perform the experiment microscopically on the individual particle level, we employed an optical micromanipulating system that is similar to the one described in Ref. 26. The experiment setup used in our experiment is schematically shown in Fig. 1. A semiconductor laser of 780 $\mathrm{nm}$ is used as the light source of the optical tweezers. The laser beam is introduced into an inversed microscope, reflected by a dichromatic mirror, which is inserted in the microscope, and incident upon a high-numerical-aperture (NA1.35) oil-immersion microscope objective, which focuses a strongly convergent beam into a glass sample cell. Due to optical momentum transfer to the particle suspended in the solution in the cell, the strongly convergent beam will exert a force on the particle and hold the particle at a point near its focus. Three-dimensional motion of the sample stage with an adjustable moving speed is controlled by a computer. To trap a particle is accomplished by moving the microscope sample stage. An electronic shutter in the laser beam path is used to control the duration of particles held in the optical trap.

For a suspension, we used polystyrene (PS) spheres with a radius of $a_{0}=0.497 \pm 0.011 \mu \mathrm{m}$ (a commercial product with Catalog number 4009A, from Duke Scientific Corporation, USA.). The number concentration of PS particles in the 
TABLE I. Sticking probability $(P)$, stability ratio $(W)$, and zeta potential vs concentrations of $\mathrm{NaCl}\left(C_{\mathrm{NaCl}}\right)$. All experiments were performed at $20^{\circ} \mathrm{C}$.

\begin{tabular}{lcccccc}
\hline \hline$C_{\mathrm{NaCl}}\left(\mathrm{mol} \mathrm{L}^{-1}\right)$ & 0 & 0.05 & 0.1 & 0.15 & 0.20 & 0.3 \\
\hline$p(\%)$ & 0 & $10( \pm 4)$ & $22( \pm 4)$ & $60( \pm 8)$ & $\sim 100$ & 100 \\
$p_{\text {macro }}(\%)$ & 0 & $11( \pm 5)$ & $20( \pm 5)$ & $55( \pm 6)$ & $90( \pm 3)$ & 100 \\
$W$ & $\sim \infty$ & $10( \pm 4)$ & $4.6( \pm 0.8)$ & $1.7( \pm 0.2)$ & $\sim 1$ & 1.00 \\
$W_{\text {macro }}$ & $\sim \infty$ & $9( \pm 4)$ & $5( \pm 0.2)$ & $1.8( \pm 0.2)$ & $1.10( \pm 0.04)$ & 1.00 \\
Zeta potential $(\mathrm{mV})$ & $-54( \pm 1)$ & $-49( \pm 1)$ & $-36( \pm 5)$ & $-32( \pm 3)$ & $-21( \pm 6)$ & $-19( \pm 4)$ \\
\hline \hline
\end{tabular}

final solution in our experiments was $1.0 \times 10^{8} \mathrm{~cm}^{-3} . \mathrm{NaCl}$, serving as the electrolyte, was added to induce colloidal coagulation. Change in the total potential energy of the interaction between the particles was attained by adjusting the concentration of $\mathrm{NaCl}$.

Artificial collisions were induced with the following procedure. First we used optical tweezers to catch one particle and then the second. After holding this pair of particles together in the optical trap for certain duration to induce collisions, we released them from the trap and traced them to check if they remained together or separated. Each time we test one particle pair for its collision outcome.

After sampling $n$ pairs of particles for such artificial collisions, we can get a sticking probability by calculating the ratio of the number of sticking pairs $n_{c}$ to $n$ using Eq. (2). This procedure was repeated at different levels of interaction force between particles by adjusting the electrolyte $(\mathrm{NaCl})$ concentration $\left(C_{\mathrm{NaCl}}\right)$. For each electrolyte concentration, over ten groups of independent experiments were performed; and for each experiment, 100 pairs of particles were sampled.

To examine the possible influence of the trapping speed on our results, we roughly measured its magnitude using the following method. We gradually moved the optical trap to approach a particle, and recorded the process of the particle being trapped into the trapping center in video recorder. When playing back the process, we can measure both the length it traveled and the time spent moving through the distance. Assuming that the particle attracted to the optical trap experiences a constant acceleration, we can readily estimate the particle's final speed as a rough estimate of the trapping speed. By changing the level of laser power, the trapping speed can be changed to a certain extent.

To minimize the influence of the trapping speed on the results, we used the lowest possible laser power, just enough to catch and hold two particles in the trap for our artificially induced collision experiments.

To compare the stability ratios $W$ obtained through the microscopic approach with those from the macroscopic approach (denoted by $W_{\text {macro }}$ ), based on Eq. (1), we measured the relative coagulation rates $k_{\text {rapid }}$ and $k$, coming from the slopes of turbidity vs time function. ${ }^{9}$

Zeta potential is a good index of the magnitude of the repulsive interaction between colloidal particles and is commonly used for assessing the stability of colloidal systems (see, e.g., Ref. 4). To compare our data with zeta potential measurements, we have performed a series of zeta potential measurements with BI-ZetaPALS (Brookhaven Instrument
Corp., USA). Zeta potentials were calculated from the measured electrophoretic mobilities using the tabulated values of Ottewill and Shaw, ${ }^{27}$ which have been corrected for relaxation and retardation effects.

\section{RESULTS AND DISCUSSION}

Using the procedure described in Sec. III, we carried out a series of measurements for the accumulated sticking probabilities $(P)$, which were statistically obtained over ten groups. All sticking probabilities $(p)$, derived from Eq. (10) (by taking $\tau^{\prime}=1 \mathrm{~s}$ and $\tau^{\prime \prime}=2 \mathrm{~s}$ for $P$ ) and stability ratios $(W=1 / p)$ under different concentrations of $\mathrm{NaCl}\left(C_{\mathrm{NaCl}}\right)$, are listed in Table I.

For purpose of comparison, all data obtained through the microscopic approach, those from the macroscopic approachof Eq. (1) by the turbidity measurements ( $W_{\text {macro }}$ is the stability ratio and $p_{\text {macro }}=1 / W_{\text {macro }}$ is the sticking probablity) and zeta potentials are also listed in Table I.

\section{A. The dependency of the sticking probability $P$ on the trapping duration $\tau$}

As a typical example, the curve of the sticking probability $P$ versus $\tau$ for $C_{\mathrm{NaCl}}=0.10 \mathrm{~mol} \mathrm{~L}^{-1}$ is shown in Fig. 2 . This curve, showing exponential growth of $P$ with $\tau$, is basically consistent with Eq. (5) and the curve corresponding to Eq. (4) shown in Fig. 3 can be approximately fitted by a straight line. Therefore the correctness of the physical description using Eqs. (2)-(4) for a pair of particles (under the relaxed status) in the optical trap was verified by our experiments.

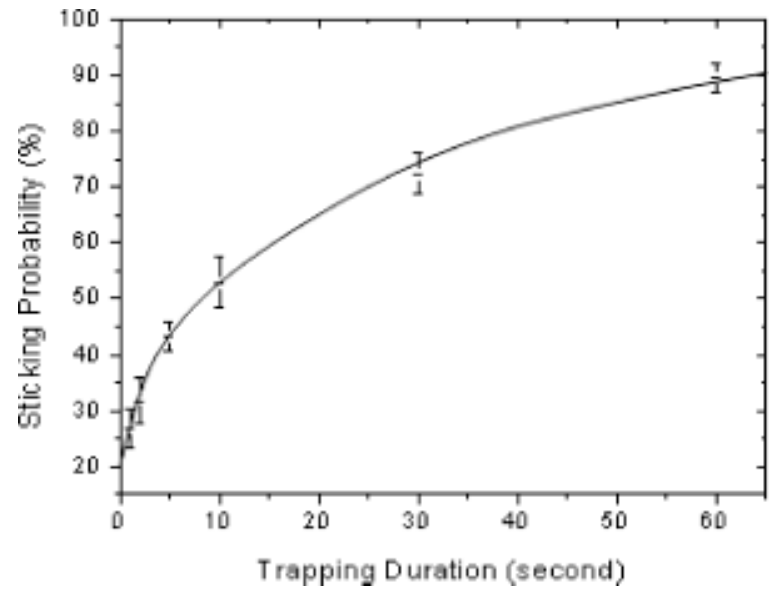

FIG. 2. The dependency of the sticking probability $P$ on the trapping duration $\tau$. (The concentration of $\mathrm{NaCl}$ is $0.1 \mathrm{M}$.) 


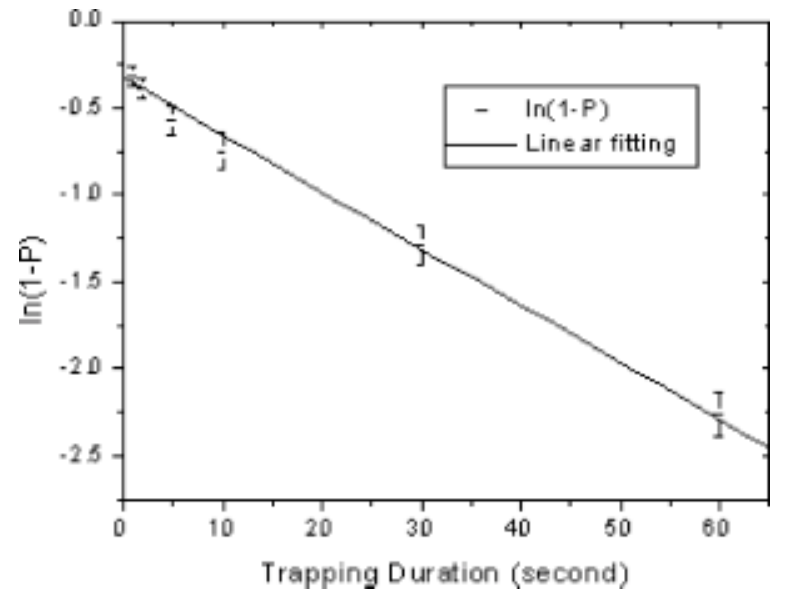

FIG. 3. $\ln (1-P)$ vs trapping duration and the linear fitting. The concentration of $\mathrm{NaCl}$ is $0.1 \mathrm{M}$.

However, the value $P(\tau=1 \mathrm{~s})$ is inconsistent with the rest of the curve: there is a great jump at the beginning of the induced collision. According to the data of $P(\tau=1 \mathrm{~s})$ $\approx 27 \%$ and $P(\tau=2 \mathrm{~s}) \approx 32 \%$, we can see that during the first of the second trapping duration, $27 \%$ of particle pairs get coagulated; but for the duration between $\tau=1 \mathrm{~s}$ and $\tau=2 \mathrm{~s}$, only about $5 \%$ more of them adhering. If one looks at the average increasing rates for the sticking probabilities for the first and the second second trapping durations, they are $27 \% / \mathrm{s}$ and $7 \% / \mathrm{s}$, respectively. Using Eq. (6) and letting $p$ $\approx p_{\text {macro }} 20 \%$, on average we have $f_{r} \approx 1.35 / \mathrm{s}$ and $0.35 / \mathrm{s}$ during the first second and the second second, respectively.

We believe that this difference corresponds to the status change from the compact to relaxed status. At the beginning of the first second trapping duration the trapping speed brings the particle pair into the compact status with higher collision frequency and then falls into the relaxed status with lower collision frequency. As a result, the collision frequency goes up first and then comes down to normal. This change in the collision frequency can account for an abnormal increasing rate of the sticking probability $P$ that appeared at the beginning and then came down to a smaller, normal increasing rate corresponding to the relaxed status. The actual collision frequency of particle pair in the compact status may be very high during very short periods of the compact status, but our time resolution is low.

\section{B. The influence of the trapping speed}

As analyzed in Sec. II, we suppose that the trapping speed is negligible in comparison to the speed associated with thermal motions. To test this argument, the sticking probabilities $P$ were measured with the artificially induced collisions at different trapping speeds. When the trapping speed increased from 20 to $55 \mu \mathrm{m} / \mathrm{s}$, we found that $P$ changed from $27( \pm 4) \%$ to $28( \pm 4) \%$ for $C_{\mathrm{NaCl}}$ $=0.10 \mathrm{~mol} \mathrm{~L}^{-1}$. The increase of the speed from 20 to 55 $\mu \mathrm{m} / \mathrm{s}$ corresponds with a gain in the particle kinetic energy by more than 7 times. If it were the kinetic energy associated with the trapping speed that played a determinative role in whether a collision is successful for particles to adhere, the great gain (by 7 times more) in kinetic energy should have caused a significant increase in the sticking probability. Apparently the above-mentioned test has shown that this is not the case; therefore the trapping speed does not essentially change the nature of collisions powered by thermal motions.

\section{Results under different electrolyte concentrations}

First we looked at the two extreme cases: when the potential barrier is very low (even negative, namely attraction) or very high in comparison with the particle thermal energy $\left(k_{B} T\right)$. There is either no resistance or very strong resistance for two particles to adhere.

When $C_{\mathrm{NaCl}}=0$, the large magnitude of zeta potential implies strong repulsion between particles. In this case the potential barrier is very high, and all pairs of colliding particles would be bounded back upon release without exception. In this case, we obtained $P \approx 0$ or $W \gg 1$ (denoted by $\left.W_{\propto}\right)$ and the system is in highly dispersed status.

On the other hand, at high electrolyte concentrations $\left(C_{\mathrm{NaCl}} \geqslant 0.3 \mathrm{~mol} \mathrm{~L}^{-1}\right)$ repulsion is fully suppressed. The particles effectively adhere to each other at almost every encounter, accordingly our data are $P \approx 1$ or $W \approx 1$. Therefore the system would be in rapidly coagulated status. In addition, in these extreme cases, the standard deviations of $P$ are very small, and $W$ are consistent with $W_{\text {macro }}$ (with the degree of repulsion between particles indicated by zeta potential values).

By adjusting the trapping duration and the trapping speed, our experiments show: $P$ remains essentially constants $\left(\approx 1\right.$ for $C_{\mathrm{NaCl}}=0 \mathrm{~mol} \mathrm{~L}^{-1}$ and $\approx 0$ for $C_{\mathrm{NaCl}}$ $\geqslant 0.3 \mathrm{~mol} \mathrm{~L}^{-1}$ ) for trapping durations ranging from 1 to $3 \mathrm{~s}$ and also for doubly raised trapping speeds. That means the artificial factors in our procedure ("trapping duration" and "trapping speed") basically have no influence on the results because particle pairs adhere or separate, regardless of whether "trapping duration" and "trapping speed" are somewhat larger or smaller.

When $\quad C_{\mathrm{NaCl}}=0 \mathrm{~mol} \mathrm{~L}^{-1}, \quad P(\tau=1)=P(\tau=2)=p=0$ and there is no difference with the macroscopic measurement.

When $C_{\mathrm{NaCl}}=0.05 \mathrm{~mol} \mathrm{~L}^{-1}$, the accumulated sticking probabilities $P$ vary very slowly with $t$, so $P(\tau=1) \approx P(\tau$ $=2$ ), from Eq. (10) we have $p \approx P(\tau=1)=10 \%$ and $W$ $=10$. Compared with the turbidity measurement, $P_{\text {macro }}$ $=11 \%$ and $W_{\text {macro }}=9$, they are in good agreement.

By using Eq. (10), for $C_{\mathrm{NaCl}}=0.1 \mathrm{~mol} \mathrm{~L}^{-1}(W \approx 5$ or $p$ $\approx 20 \%$ ) case, from the data $P_{\text {micro }}(\tau=1) \approx 27 \%$ and $P_{\text {micro }}(\tau=2) \approx 32 \%$, so we obtain $p \approx 22 \%$ or $W \approx 4.6$; against $P_{\text {macro }}=20 \%$ (or $W_{\text {macro }}=5$ ) from turbidity measurement.

For $C_{\mathrm{NaCl}}=0.15 \mathrm{~mol} \mathrm{~L}^{-1}(W \approx 1.8$ or $p \approx 55 \%)$ case, from the data $P(\tau=1) \approx 68 \%$ and $P(\tau=2) \approx 76 \%$, we have $p \approx 60 \%$ or $W \approx 1.7$; against $P_{\text {macro }}=55 \%$ (or $W_{\text {macro }}=1.8$ ).

In $C_{\mathrm{NaCl}}=0.20 \mathrm{~mol} \mathrm{~L}^{-1}$ case, using Eq. (10), in Table I we still list $p \sim 1.00$ (or $W \sim 1$ ) but this value is questionable, showing a large deviation from the turbidity measurement. Actually, because $P(\tau=1) \approx P(\tau=2) \approx 100 \%$ in our experiment, Eq. (10) has lost its capability to correct the error due to the long trapping duration. In this case, Eq. (10) becomes 
$p \approx P(\tau=1)$ and therefore can yield only an accumulated sticking probability (for multicollisions) during one second trapping. We assume this situation should be improved by choosing shorter trapping duration.

For $C_{\mathrm{NaCl}}=0.30 \mathrm{~mol} \mathrm{~L}^{-1}$ case, in Table I $p \sim 1.00$ (or $W \approx 1$ ) is also listed but we are confident of this value because of the additional information from our observation: at high $C_{\mathrm{NaCl}}\left(\geqslant 0.30 \mathrm{~mol} \mathrm{~L}^{-1}\right.$ ), even very brief contact (or even close approach) of two particles was enough for them to stick firmly together. We found that $P=100 \%$ constantly for trapping duration ranging from $\tau$ very small (smaller than 0.3 s) to $\tau=1 \mathrm{~s}$. But for the $C_{\mathrm{NaCl}}=0.20 \mathrm{~mol} \mathrm{~L}^{-1}$ case, $P$ varies from $92 \%$ to $100 \%$ corresponding to trapping duration changing from $\tau$ very small to $\tau=1 \mathrm{~s}$. This additional information implies that the energy barrier totally disappears for $C_{\mathrm{NaCl}}=0.30 \mathrm{M}$ so every collision becomes effective for aggregation; while for $C_{\mathrm{NaCl}}=0.20 \mathrm{M}$, the barrier is lower but still exists, so not every collision is effective and longer trapping causes more collisions resulting in an increase of $P$ with trapping duration.

We can see when $C_{\mathrm{NaCl}}=0,0.05,0.10,0.15$, and 0.3 , the data obtained from our microscopic approach are in good agreement with those by the turbidity measurement.

Our data are statistically reproducible as long as the experimental conditions (such as the concentrations of $\mathrm{NaCl}$ and temperature) are kept the same. At least they correctly reflect the trend that the sticking probability increases with increasing electrolyte concentration, and therefore provide useful information (like zeta potential) for judging the colloidal stability.

When taking into the trapping duration correction by using Eq. (10), we can see that our data are in good agreement with those from the turbidity measurements when the stability ratio is greater than 1.8 for $W>1.8$ (or $p<55 \%$ ) case (corresponding to the concentration of $\mathrm{NaCl}$ ranging from 0 to $0.15 \mathrm{~mol} \mathrm{~L}^{-1}$ ), as listed in Table I.

In conclusion, the microscopic approach works effectively for the DLCA region and for RLCA region with the limitation that the stability ratio is greater than 1.8 . The current difficulty for further extension of its applicable range is associated with determining the exact moment when the captured particles start to make "contact," and controlling the exact time for releasing them within a very short trapping duration.

\section{A comparision of the microscopic approach and other methodologies}

Zeta potential is also based on the nature of particle interaction for predicting dispersion stability. If the repulsion between particles indicated by zeta potential measurement is higher than particle thermal energy $\left(\sim k_{B} T\right)$, the system is stable. Usually, when the absolute value of the zeta potential is above a certain limit (say, $20 \mathrm{mV}$ ), the dispersions are considered stable. However, other factors, such as steric repulsion, may make the problem complicated. Because zeta potential is only a measure of the electrostatic interaction between individual particles; it is inadequate for supplying any information of steric hindrance. In contrast, since our approach is to bring particles together directly and to observe what actually occurs, judgment based on this process is more reliable. In this regard, our approach is apparently effective not only for electrostatic, but also for steric repulsion. An additional advantage of our procedure is that only a small amount of dispersion solution is needed for sampling tests (about $0.1 \mathrm{ml}$ ).

One major superiority of the microscopic approach as compared to macroscopic ones is that the former needs only to perform measurement, on the suspension sample itself, but the latter needs not only the sample itself for $k$ evaluation, but also one has to prepare an additional sample to acquire $k_{\text {rapid }}$. To an unknown suspension this is not always easy to do. For instance, the methods using Eq. (1) cannot, in general, be applied to the sterically stabilized systems.

\section{CONCLUSION}

An alternative approach to the study of colloidal stability at individual particle levels, by means of observing artificially induced collision, is proposed in this work. We presented a physical model that divides the artificial collisions into two statuses: compact and relaxed. We further assume that only single collision takes place in the compact status, which has much higher collision frequency and therefore primarily contributes to the sticking probability. The distinction of these two contributions - the single collision and that of multicollisions-makes it possible to evaluate the commonly accepted sticking probabilities, and therefore the stability ratios. Derived from the physical model, sticking probabilities can be statistically evaluated using data collected from the observation of artificially induced collisions. This approach would be the first experimental attempt to determine the stability ratios of a colloidal system based on Eq. (2). Although the approach is rather preliminary and requires further improvement, data thus obtained have correctly shown the trend that the sticking probability increases as the concentration of electrolyte increases; and therefore provide useful information for determining colloidal stability.

For fully dispersed $(W \gg 1)$ or rapid-coagulated ( $W$ $\approx 1$ ) systems, this approach conveniently provides a definite judgment regarding stability. For general slow-coagulated systems, the approach is shown to be valid as long as $W$ $>1.8$ (or $p<55 \%$ ). When the stability ratio is less than 1.8 , application of the approach is currently limited by the possibility of imprecise control within the shorter trapping duration.

In contrast to commonly used approaches rooted in Eq. (1) and zeta potential measurement, this microscopic approach has the capability to treat not only electrostatically but also sterically stabilized systems. Moreover, a visual understanding of the interaction between particles and of the aggregation phenomenon can be gained in addition to aiding the evaluation of stability ratios.

\section{ACKNOWLEDGMENTS}

This work is supported by Grant No. 20273065 from the National Natural Science Foundation of China and the "chuang-xin project" of Chinese Academy of Sciences. 
${ }^{1}$ H. Helmut, U. E. Stefan, B. Michal, S. Peter, and S. Hans, Langmuir 12, 5541 (1996).

${ }^{2}$ S. Hans and S. Klaus, Coagulation Kinetics and Structure Formation (VEB Deutscher Verlag der Wissenschaften, Berlin, 1987).

${ }^{3}$ J. Th. G. Overbeek, Colloid Science (Elsevier, Amsterdam, 1952), Vol. 1.

${ }^{4}$ R. J. Hunter, Zeta Potential in Colloid Science (Academic, London, 1981).

${ }^{5}$ R. H. Ottewil and J. N. Shaw, Discuss. Faraday Soc. 42, 154 (1966).

${ }^{6}$ H. Reerink and J. Th. G. Overbeek, Discuss. Faraday Soc. 18, 74 (1954).

${ }^{7}$ J. W. Lichtenbelt, H. J. M. Ras, and P. H. Wiersema, J. Colloid Interface Sci. 46, 522 (1974).

${ }^{8}$ J. W. Lichtenbelt, H. J. M. Ras, and P. H. Wiersema, J. Colloid Interface Sci. 49, 281 (1974).

${ }^{9}$ Z. W. Sun and R. L. Qiao, J. Colloid Interface Sci. 223, 126 (2000).

${ }^{10}$ A. Lips, C. Smart, and E. Willis, J. Chem. Soc., Faraday Trans. 67, 2979 (1971).

${ }^{11}$ A. Lips and E. Willis, J. Chem. Soc., Faraday Trans. 1 69, 1226 (1973).

${ }^{12}$ D. Giles and A. Lips, J. Chem. Soc., Faraday Trans. 1 74, 733 (1978).

${ }^{13}$ G. R. Zeichner and W. R. Schowalter, J. Colloid Interface Sci. 71, 236 (1979).

${ }^{14}$ B. E. Novich and T. A. Ring, J. Chem. Soc., Faraday Trans. 1, 1455 (1985).

${ }^{15}$ J. W. Virden and J. C. Berg, J. Colloid Interface Sci. 149, 528 (1992).
${ }^{16}$ M. B. Einarson and J. C. Berg, J. Colloid Interface Sci. 155, 65 (1993).

${ }^{17}$ R. Amal, J. R. Coury, J. A. Raper, W. P. Walsh, and T. D. Waite, Colloids Surf. 46, 1 (1990).

${ }^{18}$ M. Y. Han, H. K. Lee, D. F. Lawler, and S. I. Choi, Wat. Sci. Tech. 36, 69 (1997).

${ }^{19}$ M. Y. Han and H. K. Lee, Colloids Surf., A 202, 23 (2002).

${ }^{20}$ M. Mellema, J. H. J. van Opheusden, and T. van Vliet, J. Chem. Phys. 111, 6129 (1999).

${ }^{21}$ A. Ashkin, Phys. Rev. Lett. 24, 156 (1970).

${ }^{22}$ A. Ashkin, J. M. Dziedzic, J. E. Brorkholm, and S. Chu, Opt. Lett. 11, 288 (1986).

${ }^{23}$ S. M. Block, in Optical Tweezers: A New Tools for Biophysics, Noninvasive Techniques in Cell Biology, Modern Cell Biology series, edited by S. Grinstein and K. Foskett (Wiley-Liss, New York, 1990), Vol. 9.

${ }^{24} \mathrm{~J}$. Dai and M. P. Sheetz, Methods in Cell Biology: Laser Tweezers in Cell Biology (Academic, New York, 1998).

${ }^{25}$ Z. W. Sun, R. Z. Zhu, Y. M. Li, S. H. Xu, and L. R. Lou, ACTA Phys.Chim. Sin. (to be published).

${ }^{26}$ Z. W. Sun, Y. M. Li, S. H. Xu, L. R. Lou, G. L. Dai, and X. Q. Dong, J. Colloid Interface Sci. 242, 158 (2001).

${ }^{27}$ R. H. Ottewill and J. N. Shaw, J. Electroanal. Chem. Interfacial Electrochem. 37, 133 (1972). 